\title{
Impact of the energy-based and location-based LEACH secondary cluster aggregation on WSN lifetime
}

\begin{abstract}
ABSTRCT
The improvement of sensor networks' lifetime has been a major research challenge in recent years. This is because sensor nodes are battery powered and may be difficult to replace when deployed. Low energy adaptive clustering hierarchical (LEACH) routing protocol was proposed to prolong sensor nodes lifetime by dividing the network into clusters. In each cluster, a cluster head $(\mathrm{CH})$ node receives and aggregates data from other nodes. However, $\mathrm{CH}$ nodes in LEACH are randomly elected which leads to a rapid loss of network energy. This energy loss occurs when the $\mathrm{CH}$ has a low energy level or when it is far from the BS. LEACH with two level cluster head (LEACH-TLCH) protocol deploys a secondary cluster head $(2 \mathrm{CH})$ to relieve the cluster head burden in these circumstances. However, in LEACHTLCH the optimal distance of $\mathrm{CH}$ to base station (BS), and the choicest $\mathrm{CH}$ energy level for the $2 \mathrm{CH}$ to be deployed for achieving an optimal network lifetime was not considered. After a survey of related literature, we improved on LEACH-TLCH by investigating the conditions set to deploy the $2 \mathrm{CH}$ for an optimal network lifetime. Experiments were conducted to indicate how the $2 \mathrm{CH}$ impacts on the network at different $\mathrm{CH}$ energy levels and (or) $\mathrm{CH}$ distance to BS. This, is referred to as factor-based LEACH (FLEACH). Investigations in FLEACH show that as $\mathrm{CHs}$ gets farther from the $\mathrm{BS}$, the use of a $2 \mathrm{CH}$ extends the network lifetime. Similarly, an increased lifetime also results as the $\mathrm{CH}$ energy decreases when the $2 \mathrm{CH}$ is deployed. We further propose FLEACH-E which uses a deterministic $\mathrm{CH}$ selection with the deployment of $2 \mathrm{CH}$ from the outset of network operation. Results show an improved performance over existing state-of-the-art homogeneous routing protocols.
\end{abstract}

Keyword: Energy-efficient; Network lifetime; FLEACH; Wireless sensor networks; Energy aware protocols; Classification of protocols; Survey. 